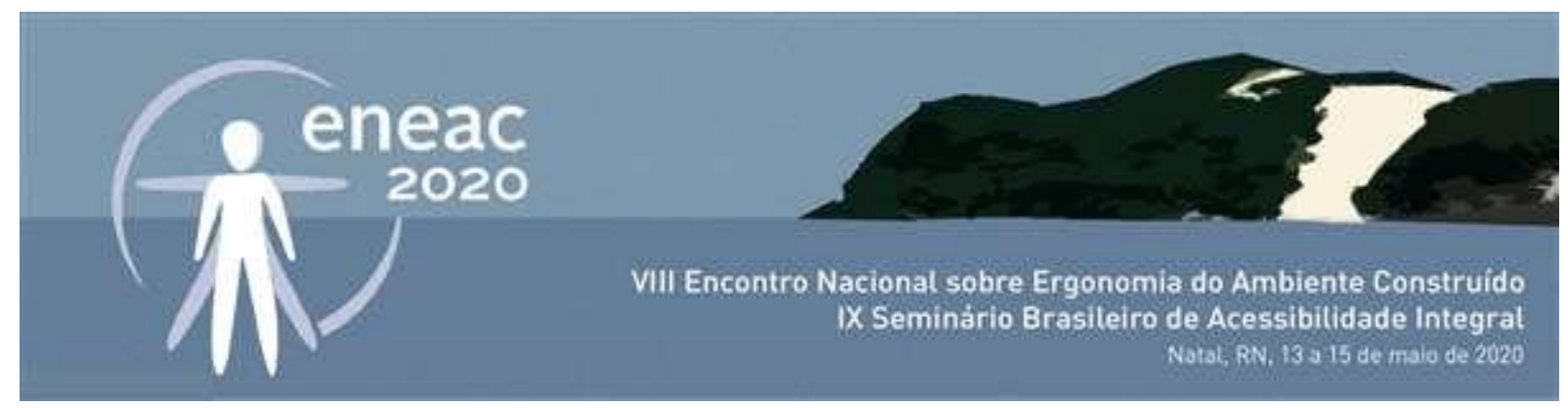

\title{
Ferramentas para a mobilidade de pessoas com deficiência visual: Revisão Sistemática de Literatura
}

\author{
Tools for mobility of visually impaired people: a Systematic \\ Literature Review
}

ESTER RODRIGUES DA COSTA JORGE

Doutoranda em Design UFPE, UNIFANOR, arq.estercosta@gmail.com

ZILSA MARIA PINTO SANTIAGO

Doutora, PPGAUD/ Universidade Federal do Ceará, zilsa@arquitetura.ufc.br

VILMA VILLAROUCO

Doutora em Engenharia, UFPE - PPGDESIGN/PPERGO, UFC -PPGAU+D, vvillarouco@gmail.com

\section{RESUMO}

As ferramentas de tecnologia assistiva, informação e comunicação têm se configurado como fortes aliadas para o incremento da mobilidade urbana no cotidiano dos cegos. Nesta direção, este artigo apresenta uma Revisão Sistemática de Literatura (RSL), que objetivou identificar metodologias, ferramentas e métodos utilizados para melhorar e facilitar os deslocamentos dessas pessoas. A pesquisa foi realizada na base de Periódico da CAPES (Scopus, Springer e Web of Science), buscando os artigos dos últimos 5 anos em idiomas inglês e português, tendo como palavras-chave: blind (cegos), tactile maps (mapas táteis), app mobility (aplicativo de mobilidade), mobility (mobilidade), spatial orientation (orientação espacial). Com a combinação destes termos, chegou-se ao total de 8 strings de busca. Assim, foram encontrados 38.492 artigos, os quais passaram por sucessíveis análises e seleções, concluindo em 12 artigos relevantes. Os resultados encontrados foram satisfatórios, pois apontaram para a importância da temática em questão, ratificando diversas pesquisas que objetivam corroborar para o desenvolvimento da mobilidade de cegos com as ferramentas estudadas.

PALAVRAS-CHAVE: cegos; mobilidade; mapas táteis; aplicativo de mobilidade; orientação espacial

\section{ABSTRACT}

Assistive technology, information and communication tools have been configured as strong allies to increase urban mobility in the daily lives of the blind. In this direction, this study aims to conduct a systematic literature review (RSL) to identify methodologies, tools and methods used with this theme. The research was conducted in the CAPES journal (Scopus, Springer and Web of science), searching the articles of the last 5 years in English and Portuguese. Keywords were used: blind, tactile maps, app 


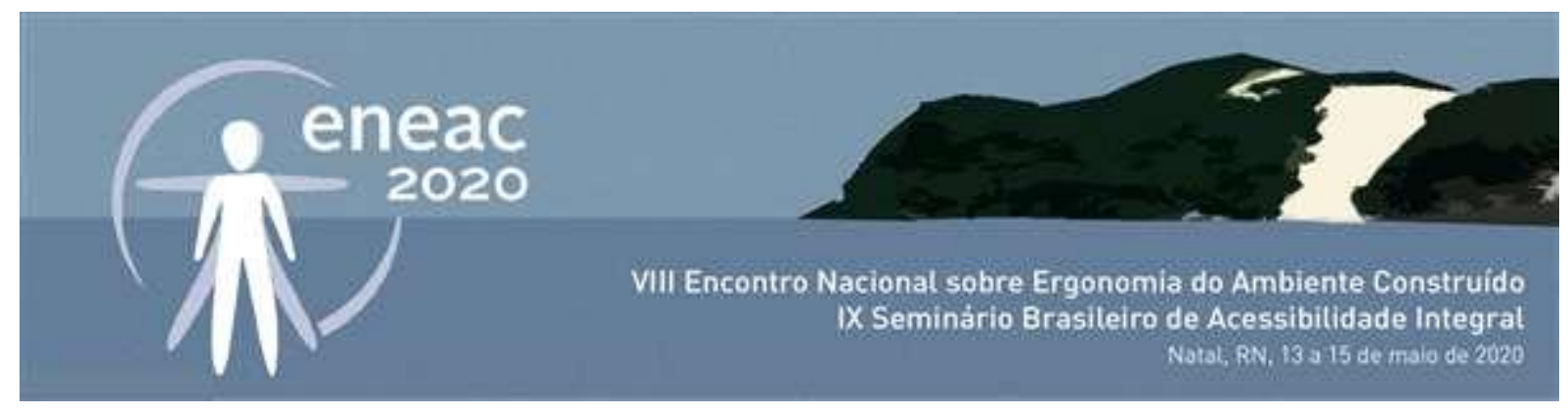

mobility, mobility, spatial orientation. By combining these terms, we reached eight search strings. Thus, 38,492 articles were found, which underwent successive analyzes and selections, culminating in 12 articles. The results were satisfactory, as they pointed to the importance of the theme in question, confirming several researches that aim to corroborate the development of blind mobility with the studied tools.

KEYWORDS: blind; mobility; tactile maps; mobility application; spatial orientation

\section{INTRODUÇÃO}

No Brasil, um dos principais direitos fundamentais que é o de ir e vir, não é alcançado pela maioria das pessoas com deficiência. Tornar um local acessível é promover condições de utilização com segurança e autonomia, independentemente de ser total ou assistida. Esta acessibilidade e mobilidade abrangem mobiliários e equipamentos urbanos, edificações, transportes, dispositivos, sistemas e meios de comunicação e informação. Entre pessoas com deficiência, o deficiente visual, é o que mais sofre exclusão na ocupação e uso dos espaços da cidade, pois o sentido da visão é reconhecidamente o mais importante canal para a aquisição da informação espacial.

Segundo Porter (2002) o deficiente visual sofre implicações psicológicas, como a irritação, stress e ansiedade, decorrentes de situações como aglomeração nas ruas e ambientes com ruído, provocando medo para se locomover. Segundo Veras e Vargas (2004), o deficiente visual, assim como as demais pessoas, passa por um processo sensorial e cognitivo, onde a recepção de informações a todo instante, lhe permite reconhecer o universo ao seu redor e o capacita para a mobilidade. Assim, percebe-se que entre as necessidades e barreiras deste tipo de usuários está a mobilidade urbana. Diante deste contexto, são utilizados recursos e estratégias denominadas de tecnologia assistiva (TA) e Tecnologias de Informação e Comunicação (TIC), objetivando melhores condições para o deficiente visual, através de uma acessibilidade efetiva, com melhor integração e apropriação nos espaços,

Diante desse contexto, para o desenvolvimento desta pesquisa foi realizado um amplo levantamento bibliográfico, através de uma revisão sistemática de literatura tendo como temática ferramentas de mobilidade como os mapas táteis e aplicativos de mobilidade urbana voltados para cegos. Nesta direção, foram revisados estudos em bases de dados pertencentes ao portal Periódicos CAPES Coordenação de Aperfeiçoamento de Pessoal de Nível Superior, objetivando identificar na literatura trabalhos, desde artigos, até dissertações e teses, que estão sendo mais relevantes para o incremento da mobilidade para cegos com o uso destas ferramentas.

\section{FERRAMENTAS DE MOBILIDADE PARA CEGOS E SUAS IMPLICAÇÕES NO COTIDIANO}

Ter o pleno funcionamento do corpo, no que concerne a fisiologia e anatomia humana é fator condicionante para a realização de atividades e participação na sociedade. Adquirir uma deficiência em qualquer etapa da vida de um indivíduo ou já nascer com ela é uma situação que pode impactar seu cotidiano, conforme preconiza a Classificação Internacional de Funcionalidade (CIF).

Segundo dados do IBGE (2010), no Brasil, entre as deficiências investigadas, a visual apresenta a maior incidência, sendo declarada por $18,8 \%$ da população, devendo-se destacar que esse percentual 


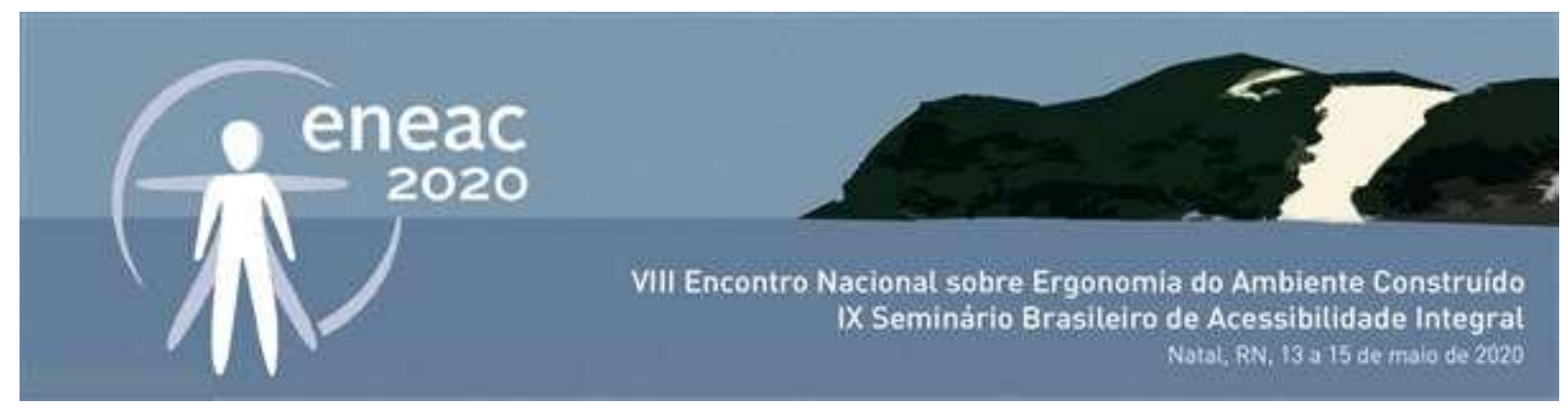

inclui desde usuários de óculos para alguma correção, até a total ausência de visão. A deficiência visual limita, e algumas vezes, até impede algumas habilidades, fazendo com que o cego se sinta marginalizado e excluído de simples ações cotidianas. Esta situação, afeta o cego e as pessoas que se relacionam com ele: familiares, amigos e outros.

"[...] dependem muitas vezes da vontade e da ação de outros para ampliar progressivamente seu conhecimento do mundo. Sua educação na busca em conseguir uma autonomia está baseada em outorgar-lhes as habilidades que lhes permitam viver na sociedade que os rodeia e a compreender suas regras." (RABASCO, 2009, p. 406).

Para se locomover, o deficiente visual precisa de algum recurso auxiliar: bengala, cão-guia, pisos táteis, semáforos com alarme sonoro, entre outros. No meio acadêmico, nas organizações ligadas a pessoas com deficiência e no mercado de produtos, a maioria desses recursos pode ser denominada de "Tecnologia Assistiva" (TA). Esta terminologia foi oficializada pelo Comitê de Ajudas Técnicas da Subsecretaria Nacional de Promoção dos Direitos da Pessoa com Deficiência e engloba produtos, recursos, metodologias, estratégias, práticas e serviços com o objetivo de promover autonomia, qualidade de vida e inclusão social.

Para Hersh e Johnson (2008), o objetivo social da tecnologia assistiva é melhorar a qualidade de vida de pessoas com deficiência, superar a lacuna entre o que essas pessoas querem fazer e o que a infraestrutura social existente permite que elas façam. Em conjunto com as tecnologias assistivas (TAs) também são utilizadas as tecnologias de informação e comunicação (TICs) através de interfaces digitais e sistemas de georeferenciamento.

Nesta direção, o desenvolvimento de ferramentas para otimizar a mobilidade urbana tem sido um objeto de estudo bastante explorado, se configurando com uma ampla gama de possibilidades de informação e consequentemente grande importância para a assistência de seus usuários. Entre estas ferramentas estão os mapas táteis e os sistemas de mobilidade georreferenciados.

\section{METODOLOGIA}

Com o propósito de atender ao objetivo inicialmente proposto, a pesquisa se inicia com uma revisão sistemática da literatura com base na Cochrane Collaboration (2013), quando coloca que a revisão sistemática é uma síntese rigorosa de pesquisas relacionadas com uma questão específica.

As revisões sistemáticas de literatura se diferem das revisões narrativas ou tradicionais, pois essas são mais amplas, com informações generalizadas sobre o tema em questão, tendo como fontes, os livros. Segundo Botelho (2013), são diferentes também das revisões integrativas, pois utilizam diferentes delineamentos em um mesmo exercício investigativo e podem constar em seu escopo a opinião do próprio autor. As revisões sistemáticas são consideradas estudos secundários, tendo nos estudos primários sua fonte de dados. Entende-se por estudos primários os artigos científicos que relatam os resultados de pesquisa em primeira mão.

\section{Revisão Sistemática de Literatura}

A Revisão Sistemática da Literatura (RSL) aqui exposta, foi iniciada em agosto de 2019. A base consultada foi a de periódicos CAPES, tendo como principal critério de escolha o considerável 


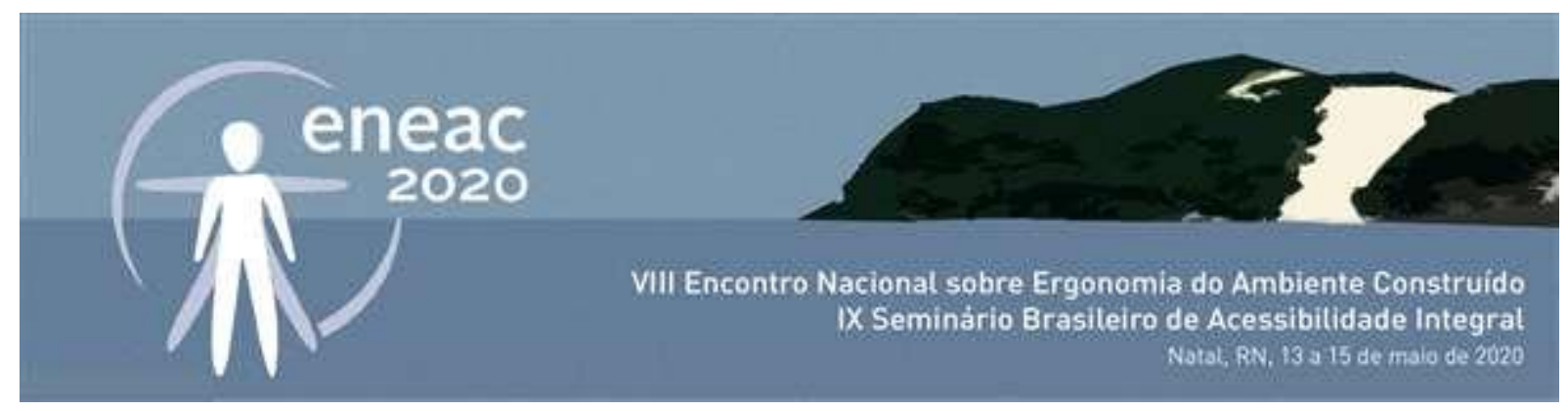

número de periódicos disponíveis na realização de suas buscas: Scopus (Elsevier), Springer (CrossRef) e Science Citation Index Expanded (Web of Science). Assim, foi seguido uma sistemática, com a descrição dos passos a seguir:

Na primeira etapa foi realizada uma Busca Avançada escolhida para artigos como: "Qualquer", ou seja, os conteúdos foram localizados pelo título, ou pelo autor, ou pelo assunto. Como filtro os idiomas inglês e português, no período dos últimos 5 anos (2015-2019). Para refinar a busca, foi adicionado também o filtro para periódicos revisados por pares, isto é, apenas para artigos avaliados por ao menos dois avaliadores.

Na segunda análise foi conduzida uma busca por tópicos e posteriormente título, resumo e conteúdo relevante. Nesta sequência pode-se chegar ao número de artigos mais relevantes para o escopo da pesquisa, identificando metodologias, ferramentas e métodos utilizados para o incremento da mobilidade dos cegos.

\section{Busca por artigos}

Nesta primeira fase do estudo, a busca concentrou-se somente em artigos do Portal de Periódicos Capes, restringindo ao Scopus (Elsevier), Springer(CrossRef) e Science Citation Index Expanded (Web of Science).

A primeira busca, retornou um total geral de 38.541 artigos, utilizando a categoria de busca por assunto, com filtro por idioma (inglês e português) e selecionados por um período dos últimos 5 anos. Com o uso 8 strings ( 4 em inglês e 4 em português) foram encontrados os seguintes resultados. A tabela 1, mostra os strings em inglês (blind+tactile maps; blind+app mobility; blind+ mobility e blind+spatial orientation) e a tabela 2 os strings em português (cegos_mapas táteis; cegos+ app de mobilidade; cegos+mobilidade; cegos+ orientação espacial).

Tabela 1: 1a Busca nos Periódicos capes - strings em inglês

\begin{tabular}{c|c}
$\begin{array}{c}\text { Categoria da busca: qualquer-qualquer } \\
\text { Palavra- chave/booleano/palavra-chave }\end{array}$ & $\begin{array}{c}\text { Resultado } \\
\left(\mathrm{n}^{\circ} \text { de artigos filtrados por pares) }\right.\end{array}$ \\
\hline Blind+Tactile Maps & 1137 \\
\hline Blind+ App Mobility & 698 \\
\hline Blind+Mobility & 18833 \\
\hline Blind+ Spatial Orientation & 9594 \\
\hline
\end{tabular}

Fonte: autoras, 2019.

Tabela 2: 1a Busca nos Periódicos capes - strings em português

\begin{tabular}{c|c}
$\begin{array}{c}\text { Categoria da busca: qualquer-qualquer } \\
\text { Palavra- chave/booleano/palavra- } \\
\text { chave }\end{array}$ & $\begin{array}{c}\text { Resultado } \\
\left(\mathrm{n}^{\circ} \text { de artigos filtrados por pares) }\right.\end{array}$ \\
\hline Cegos+ Mapas Táteis & 0 \\
\hline Cegos+ App De Mobilidade & 13 \\
\hline Cegos+ Mobilidade & 14 \\
\hline Cegos+ Orientação Espacial & Fonte: autoras, 2019.
\end{tabular}




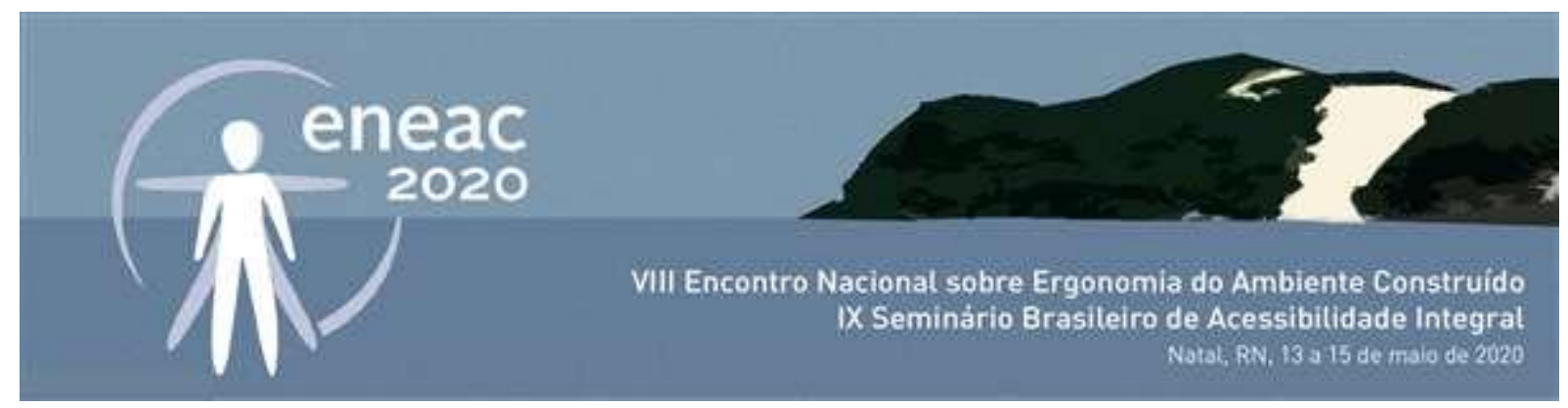

Devido ao número reduzido de artigos em português, apenas os artigos pesquisados em inglês passaram pela seleção do filtro: assunto, houve um decréscimo para 18.190 artigos. Em seguida foram selecionados pela análise dos títulos, totalizando 1.253 artigos, posteriormente foram analisados através da leitura dos resumos, resultando em 54 artigos e finalmente a leitura completa dos artigos, finalizando 13 artigos com relevância para o tema, em resumo na tabela 3.

Tabela 3: Busca refinadas nos Periódicos capes

\begin{tabular}{|c|c|c|c|c|}
\hline $\begin{array}{c}\text { Categoria da busca: } \\
\text { assunto/título/resumo/ relevância } \\
\text { Palavra- chave/booleano/palavra-chave }\end{array}$ & Assunto & Título & Resumo & Relevância \\
\hline $\begin{array}{l}\text { Blind+Tactile Maps } \\
\text { Cegos + mapas táteis }\end{array}$ & 536 & 103 & 12 & 6 \\
\hline $\begin{array}{c}\text { Blind + App Mobility } \\
\text { Cegos + app de mobilidade }\end{array}$ & 269 & 15 & 10 & 3 \\
\hline $\begin{array}{c}\text { Blind+Mobility } \\
\text { Cegos + mobilidade }\end{array}$ & 9170 & 673 & 16 & 2 \\
\hline $\begin{array}{l}\text { Blind+ Spatial Orientation } \\
\text { Cegos + orientação espacial }\end{array}$ & 3058 & 280 & 11 & 2 \\
\hline
\end{tabular}

Na última etapa, onde foram revisados 13 artigos, deu-se uma análise através do reconhecimento das temáticas elencadas com base nos strings de busca, as quais serviram para a criação de grupos temáticos para a classificação dos artigos. Os grupos são: Cegos e Mapas Táteis, Cegos e Aplicativos de mobilidade, Cegos e Mobilidade e Cegos e Orientação espacial. Os grupos estão na figura a seguir:

Figura 1: Grupos dos artigos de maior relevância
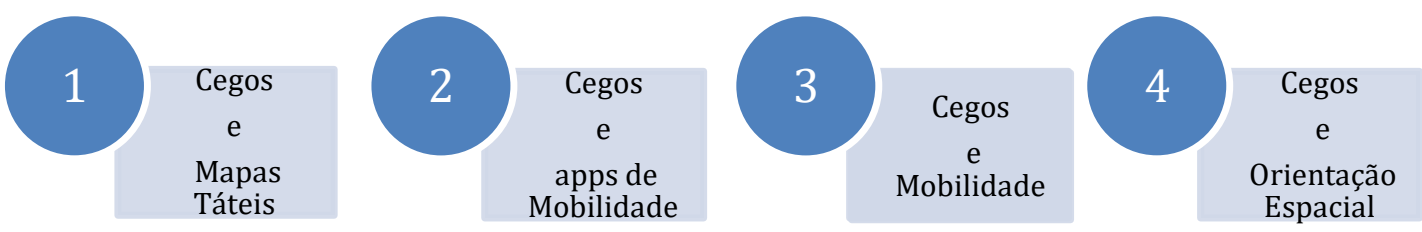

Fonte: autoras, 2019

\section{RESULTADOS}

Com o intuito de tornar viável a escrita deste estudo e conseguir abranger os artigos com maior relevância, foram selecionados apenas 13 artigos para serem apresentados e analisados nesta revisão. A apresentação se deu através dos resumos (extraído dos textos originais) adaptados e com tradução livre das autoras, com o objetivo de abranger sinteticamente as informações mais importantes para o estudo. A revisão engloba pesquisadores de diversos países: Brasil, Estados Unidos, Inglaterra, Espanha, Itália, Grécia, República Checa, entre outros. Assim, serão 


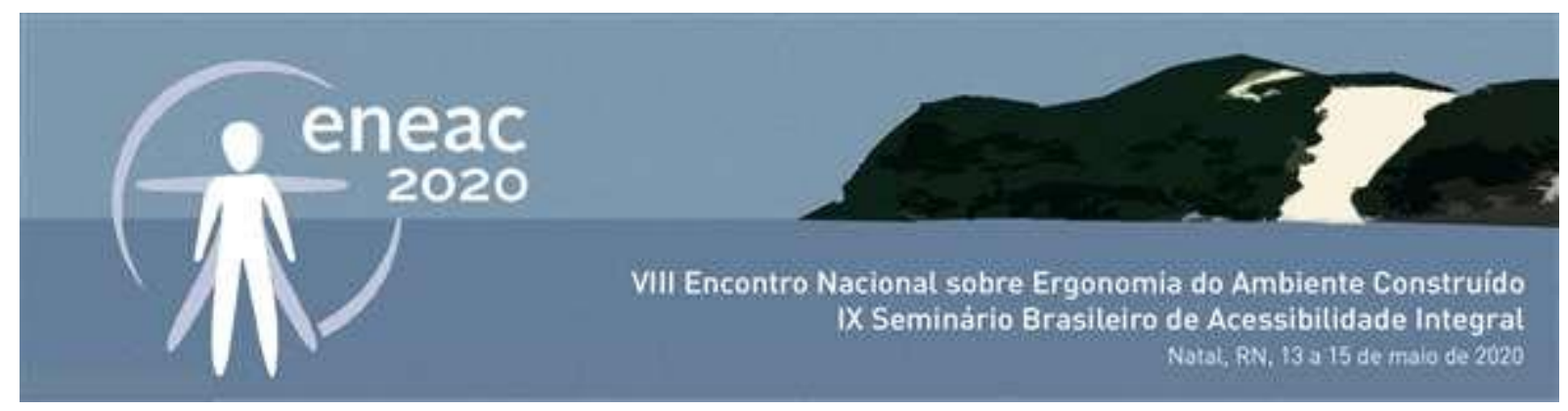

apresentados, de acordo, com a clasificação inserida nos grupos específicos apresentados na metodologia anteriormente ( Fig.01).

\subsection{Grupo 1}

Neste grupo foram analisados artigos que tiveram como abordagem concomitante as palavras-chave: cegos e mapas táteis. Assim como nos demais grupos, foram organizados com a seguintes disposição de dados: grupo do trabalho, título do trabalho, autor(es), local de origem, ano de publicação e resumo adaptado.

1. Imagens táteis tridimensionais: um modelo para a tradução tátil a partir de imagens estáticas bidimensionais - de Emilia Christie Picelli Sanches, Juliana Bueno, Claudia Mara Scudelari de Macedo. São Paulo, 2017.

Neste artigo, as autoras apresentam um modelo para tradução de imagens estáticas bidimensionais em imagens táteis tridimensionais. Para isso, partem de uma breve revisão da literatura sobre cegueira, percepção tátil e imagens táteis. Na sequência, apresentam o modelo de tradução em três partes: (1) recomendações da literatura; (2) estrutura e (3) modelo preliminar para teste. Depois, descrevem o teste do modelo realizado com dois designers com habilidades de modelagem digital (potenciais usuários).

2. Analysis of Wayfinding Strategies of Blind People Using Tactile Maps - Fátima Almeida, Laura Martins, Francisco Lima. Recife, 2015.

O estudo realizou uma pequisa através de uma rota desconhecida com a ajuda do mapa tátil exigindo do usuário não apenas entender o significado de cada textura e cada imagem de alto relevo com a ajuda do subtítulo Braille, mas também aplicar estratégias que ajudassem o desenvolvimento da representação mental do edifício a ser conhecido e a rota a ser realizada. Para tanto, houve duas estratégias que interferiram no planejamento e execução da rota: a compreensão do espaço, baseada exclusivamente no rastreamento da rota ou na percepção ambiental de todo o mapa de rastreamento. Conclui-se que as decisões foram relacionadas às estratégias de como eles usaram o mapa tátil e o mesmo determinou o desempenho do usuário. Também foi observado que três estratégias foram aplicada à leitura háptica no mapa para obter uma idéia inicial do ambiente igual àquelas usadas para o conhecimento espacial, através da identificação de parâmetros de referência, reconhecimento de rotas ou conhecimento da área. Conclui-se que a maioria teve a tendência de planejar a rota usando a mesma estratégia aplicada para entender o mapa. Devido à complexidade da tarefa de encontrar caminhos para pessoas cegas, é sugerido que o mapa tátil não seja estudado isoladamente, mas de uma visão sistêmica da ergonomia que o coloca como parte de um sistema de informação que favorece a tomada de decisões no mapa. Para esse fim, é essencial incluir parâmetros de orientação e mobilidade no início do processo de design de sistemas concomitantes de localização de caminhos para projetos arquitetônicos para tais parâmetros, para facilitar o desenvolvimento da representação mental do ambiente e das decisões de cegos que respeitam suas necessidades, habilidades e limitações, além de facilitar o planejamento e a execução de uma rota com autonomia, segurança e satisfação.

3. Movement Strategies During Haptic Search: Implications for the Learning of Blind Populations. Morash V. Berkeley, Estados Unidos da América, 2014.

Esta pesquisa desenvolvida para elaboração de dissertação, investiga as estratégias de movimento de 


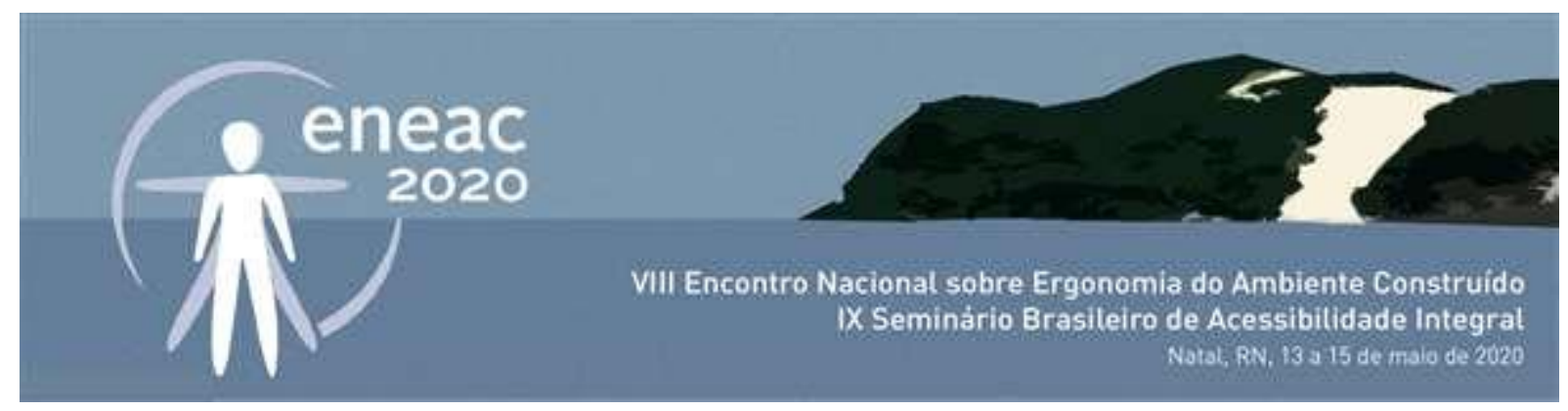

dedos usadas por participantes com visão vendada ao concluir uma tarefa de pesquisa em um mapa tátil. Esses participantes podem ser considerados semelhantes a indivíduos com perda recente da visão, por exemplo, devido a causas relacionadas à idade que são responsáveis pela maioria das deficiências visuais no mundo desenvolvido. Os resultados também podem ser estendidos a crianças com deficiência visual adquirida, que provavelmente encontrarão um grande número de mapas e gráficos táteis em sua educação continuada. Os mapas táteis e os gráficos táteis em geral oferecem oportunidades a indivíduos com pouca ou nenhuma visão de acessar materiais educacionais, profissionais e outros materiais gráficos. No entanto, o desempenho com gráficos táteis depende muito das estratégias de movimento do dedo usadas. Os resultados podem fornecer informações sobre o tipo de estratégias de movimento que provavelmente serão usadas por indivíduos com uma deficiência visual adquirida recentemente e podem informar o treinamento tátil e o design de telas táteis para esses indivíduos.

4. Landmark-enhanced route itineraries for navigation of blind pedestrians in urban environment. Balata J; Mikovec Z; Slavik P. Praga, República Checa, 2018.

Neste artigo, os autores descrevem o design de um sistema, relatando os estudos que realizaram com 43 participantes com deficiência visual. Desenvolveram um método para geração automática de instruções de navegação, aprimoradas por pontos de referência para pedestres cegos, que se baseia na criação de novas estruturas de dados GIS baseadas na calçada, desenvolvimento do algoritmo para geração de instruções de navegação em linguagem natural e algoritmo de roteamento otimizado para necessidades de viajantes com deficiência visual. Realizaram um estudo comparativo de instruções de navegação aprimoradas por pontos de referência (Landmark) com instruções de navegação baseadas em métricas (Metric) com 16 participantes com deficiência visual. Embora estudos anteriores mostrem que a navegação baseada em pontos de referência é melhor para a navegação de pedestres, os resultados medidos foram inconclusivos. No entanto, a avaliação subjetiva mostrou a preferência pela versão com pontos de referência melhorados. Além disso, forneceram um conjunto de recomendações de design para a criação de instruções de navegação relacionadas principalmente a cantos e travessias

5. On my right or on your left? Spontaneous spatial perspective taking in blind people. Tinti C; Chiesa S; Cavaglià R; Dalmasso S; Pia L;Schmidt S. Turim, Italia, 2018.

Para os autores, a tomada de perspectiva espacial é uma habilidade humana que permite assumir o ponto de vista espacial de outra pessoa. Os dados mostram que a tomada de perspectiva espacial pode surgir espontaneamente pela mera presença de outra pessoa no ambiente. Neste estudo foi investigado se esse fenômeno também é observável em pessoas cegas. Os participantes, alguns cegos e outros com os olhos vendados, exploraram um mapa tátil tridimensional e memorizaram a localização de diferentes pontos de referência. Depois, após a apresentação dos sons provenientes de três pontos de referência - posicionados à direita, à esquerda e na frente - os participantes tiveram que indicar a posição recíproca dos dois pontos de referência laterais. Os resultados mostraram que, quando o som proveniente do marco frontal sugeria a presença de uma pessoa que fala (voz) ou que se move (passos), várias pessoas cegas e com visão adotam a perspectiva dessa pessoa. Esses achados sugerem que estímulos auditivos podem desencadear perspectivas espontâneas espaciais, tanto na visão quanto na visão de pessoas cegas.

6. Cognitive load of walking in people who are blind: subjective and objective measures for assessment. Caroline Pigeona; Tong Lib; Fabien Moreau; Gilbert Pradelb,c; Claude Marin-Lamelleta. 


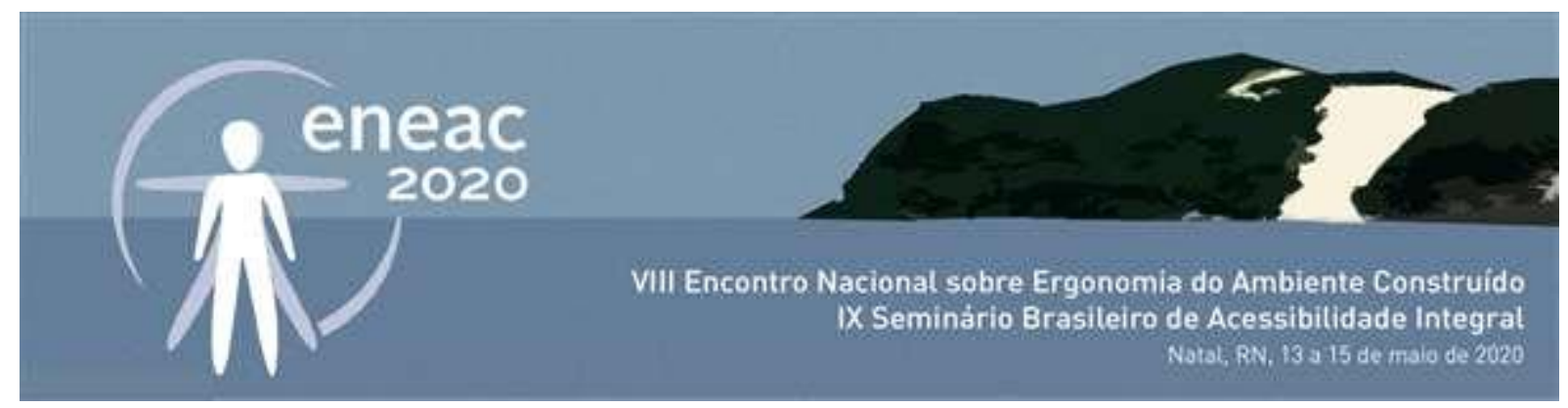

France, 2019.

O objetivo deste estudo foi avaliar a carga cognitiva provocada pelo ato de caminhar em pessoas cegas, usando a análise da marcha, um paradigma de dupla tarefa e uma avaliação subjetiva da cognição carga.Para isso, foram analisados 25 adultos cegos, os quais andaram 40 metros. Em um primeiro estudo, os participantes andaram normalmente (condição de controle). Em outro, eles caminharam enquanto realizavam um tempo de reação auditivo, tarefa simples, e no terceiro teste, eles realizaram a tarefa também simples do tempo de reação evitando obstáculos. Além do desempenho da tarefa e do tempo de reação, a velocidade de caminhada foi registrada e os participantes forneceram uma avaliação subjetiva da carga cognitiva após cada tentativa. $O$ desempenho dos participantes com menos de 60 anos foi comparado com aqueles com mais de 60 anos. Poucos efeitos significativos da idade foram encontrados.

Concluiu-se que andar reduziu o desempenho da tarefa simples de tempo de reação e aumentou a partir da avaliação subjetiva, quando indicou que a carga cognitiva aumentou quando obstáculos estavam presentes. Poucos efeitos significativos da idade foram encontrados. Andar sem visão envolve uma carga cognitiva que aumenta quando o ambiente se torna complexo. Os resultados obtidos permitiram recomendações a sugerir para o projeto de dispositivos de mobilidade tecnológica.

\subsection{Grupo 2}

Neste grupo foram analisados artigos que tiveram como abordagem concomitante as palavras-chave: cegos e aplicativos de mobilidade.

1. Blind MuseumTourer: a System for Self-Guided Tours in Museums and Blind Indoor Navigation. Meliones A; Sampson D; Piraeus. Grécia, 2018.

Neste trabalho, foi aproveitada a experiência do aplicativo de navegação para pedestres ao ar livre do smartphone BlindHelper e apresentado o Blind MuseumTourer, um sistema de navegação autônoma interativa interna, para pessoas e grupos cegos e deficientes visuais (por exemplo, alunos), em passeios auto-guiados em museus. Um protótipo piloto foi desenvolvido e está atualmente em avaliação no Museu Tactual com a colaboração do Farol para Cegos da Grécia. A publicação descreve a funcionalidade do aplicativo e avalia as possibilidades de tecnologias utilizadas em local interno. Os conceitos desenvolvidos, incluindo correspondência de mapas que é um conceito-chave para navegação interna, aplicam-se de maneira semelhante a outros casos de uso de orientação interna que envolvem locais complexos, como hospitais, shoppings, aeroportos, estações de trem, edifícios públicos e municipais, universidades, resorts, grandes navios de passageiros etc. $O$ aplicativo Android apresentado é efetivamente um sistema Blind Indoor Guide, para uma navegação em ambientes internos, precisa e confiável.

2. Evaluation of an Accessible, Real-Time, and Infrastructure-Free Indoor Navigation System by Users Who Are Blind in the Mall of America. Giudice N; Whalen W;Riehle T; Anderson S; Doore S. Estados Unidos, 2019.

O texto descreve uma avaliação do MagNav, um sistema de navegação interna sem infraestrutura e baseado em fala. A pesquisa foi realizada no Mall of America, o maior centro comercial dos Estados Unidos, para investigar empiricamente a carga de memória durante a utilização do sistema. São feitas comparações para desempenho da orientação em rota, entre o uso de instruções de rota atualizadas 


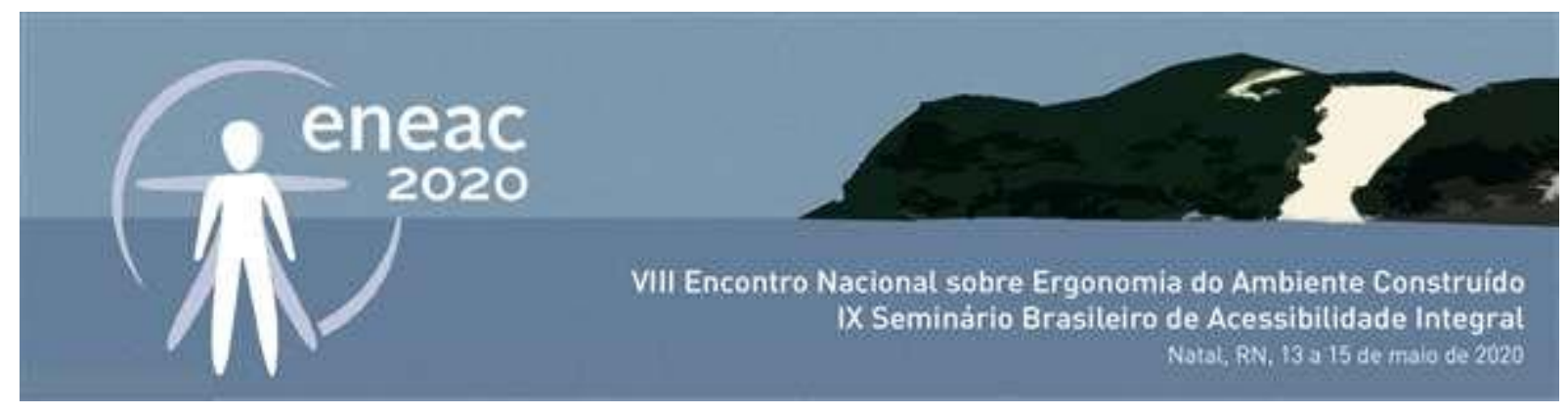

em tempo real (condição auxiliada pelo sistema) e uma assistência do sistema (condição baseada em memória) em que as mesmas instruções foram fornecidas apenas antes do percurso. Os resultados das três medidas de teste fornecem evidências comportamentais convincentes de que os navegadores cegos que recebem informações verbais em tempo real do sistema MagNav realizaram o percurso mais rápido (tempo de navegação), com mais precisão ( menos erros ao chegar ao destino) e com mais confiança (menos solicitações de assistência do espectador) em comparação às condições em que a mesma informação de rota estava disponível apenas para eles antes da viagem. Esta pesquisa fornece evidências quantitativas e qualitativas convincentes, mostrando a utilidade de um sistema de navegação de demanda sem muitos requisitos da memória e apóia a teoria de que o desempenho funcional da navegação equivalente é possível quando o acesso a informações ambientais em tempo real está disponível, independentemente do status visual. As descobertas fornecem informações sobre a importância dos desenvolvedores de sistemas de navegação acessíveis para empregar interfaces que minimizam as demandas de memória

3. Accessible smartphones for blind users: A case study for a wayfinding system Rodriguez-Sanchez M. Moreno-Alvarez M; Martin E; Borromeo S; Hernandez-Tamames J. Madri, Espanha, 2014.

Este estudo apresenta uma solução completa para gerenciar as funções básicas de um smartphone e orientar os usuários usando um aplicativo de orientação. Dessa forma, um usuário cego poderia ir trabalhar a partir de sua casa de maneira autônoma, usando um aplicativo de busca de informações adaptável em seu smartphone. $\mathrm{O}$ aplicativo wayfinding combina texto, mapa, feedback auditivo e tátil para fornecer as informações. Dezoito usuários com deficiência visual testaram o aplicativo.

Resultados preliminares deste estudo mostraram que pessoas cegas e usuários com visão limitada podem efetivamente usar o aplicativo de orientação sem ajuda. A avaliação também confirma a utilidade de estender o feedback de vibração para transmitir informações de distância e informações direcionais. A validação foi bem-sucedida para dispositivos iOS e Android.

\subsection{Grupo 3}

Neste grupo foram analisados artigos que tiveram como abordagem concomitante as palavras-chave: cegos e mobilidade.

1. A urca é o paraíso dos cegos: mobilidade urbana, acesso à cidade e territorialização. Olivia von der Weid. Rio de janeiro, 2015.

A autora expõe que no artigo foi abordada a relação de pessoas cegas com o cenário urbano do Rio de Janeiro a partir de seus deslocamentos, pelos sentidos de familiaridade ou distância atribuídos aos bairros, aos caminhos ou trajetos percorridos. Representações da cidade e de locais específicos desta cidade. Para entender como pessoas cegas desenvolvem seus percursos urbanos e as suas percepções destes lugares foram analisados manuais de formação de professores na área da deficiência visual e material de campo do Instituto Benjamin Constant (IBC). No material de campo encontram-se entrevistas com profissionais do Instituto, observação participante de atendimentos em Orientação e Mobilidade $(\mathrm{OM})$ da área de reabilitação, material do curso de formação em técnico de Orientação e Mobilidade, de 40 horas, realizado em abril de 2012 no IBC, além de entrevistas com pessoas cegas.

2. From science to technology: orientation and mobility in blind children and adults. Cuturi L; AggiusVella E; Campus C; Parmiggiani A; Gori M. Genova, Itália, 2016. 


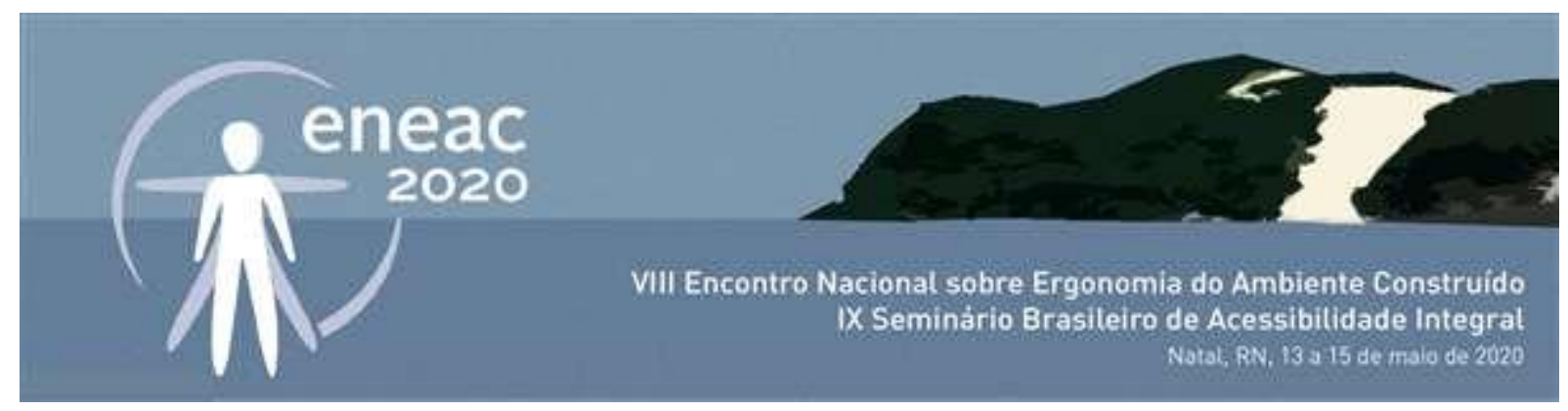

Trata do desenvolvimento de soluções tecnológicas para mobilidade de pessoas cegas e coloca que a maioria desses dispositivos foi desenvolvida sem considerar os mecanismos cerebrais subjacentes ao déficit ou a capacidade natural do cérebro de processar informações. A maioria deles usa sistemas de feedback complexos e sobrecarrega as capacidades sensoriais, atencionais e de memória. Neste artigo foram revisados os estudos neurocientíficos sobre orientação e mobilidade em adultos e crianças com deficiência visual e apresentados os dispositivos tecnológicos desenvolvidos até agora para melhorar as habilidades de locomoção. Também foram discutidos como essas soluções poderiam ser melhoradas. Para alcançar um grande número de usuários, as novas soluções tecnológicas precisam ser simples e acessíveis (por exemplo, cobertas por materiais táteis adequados e produzindo sons adequados). Tarefas diferentes dependem de informações sensoriais diferentes e é necessário identificar o melhor feedback sensorial para transmitir um certo tipo de informação e, então, melhorar esse sinal. A simplicidade do dispositivo é importante para atender às capacidades das crianças, por exemplo. Essa comunicação deve levar a uma nova maneira de desenvolver sistemas que levem em consideração i) os mecanismos cerebrais que subtendem o déficit e ii) os cognitivos atencionais, capacidades que crianças e adultos exploram para processar os sinais sensoriais fornecidos pela solução tecnológica

\subsection{Grupo 4}

Neste grupo foram analisados artigos que tiveram como abordagem concomitante as palavras-chave: cegos e orientação espacial.

1. Shape perception and navigation in blind adults. Gori M; Cappagli G; Baud-Bovy G; Finocchietti S. Milão, Itália, 2017.

No estudo apresentado neste texto foi desenvolvido um novo paradigma para testar a percepção auditiva e as habilidades de navegação em indivíduos cegos, e investigar o efeito que a experiência visual tem na capacidade de reproduzir caminhos simples e complexos. Os resultados mostram três principais deficiências específicas para indivíduos cegos precoces. A primeira é a tendência de comprimir as formas reproduzidas durante a navegação. O segundo é a dificuldade em reconhecer estímulos complexos de áudio e, finalmente, o terceiro é a dificuldade em reproduzir a forma desejada: os participantes cegos precoces ocasionalmente relatavam perceber um quadrado, mas na verdade reproduziam um círculo durante a tarefa de navegação Para concluir, nossos resultados sugerem que indivíduos cegos congênitos apresentam um déficit significativo na interpretação de sinais geométricos auditivos para navegação. O comprometimento auditivo observado neste estudo pode estar relacionado à falta de representação euclidiana, ou à persistência de um quadro de referência egocêntrico em indivíduos cegos congênitos. Uma caminhada eficiente é mediada pela integração de sinais tátil de áudio com feedback do sistema motor. Compreender como esse processo ocorre em indivíduos cegos pode melhorar o desenvolvimento de tecnologias de reabilitação com orientação científica para navegação.

2. Indoor navigation by blind people: Behaviors and challenges in unfamiliar spaces and buildings. Jeamwatthanachai W; Wald M; Wills G. Inglaterra, 2019.

Várias pessoas com deficiência visual sofrem com atividades relacionadas à navegação devido a contratempos que as desencorajam a sair para atividades e interações sociais. Ao contrário do exterior, viajar dentro de espaços públicos é uma história diferente, pois muitas dicas ambientais não podem ser usadas e têm seu próprio conjunto de dificuldades. Algumas tecnologias foram admitidas 


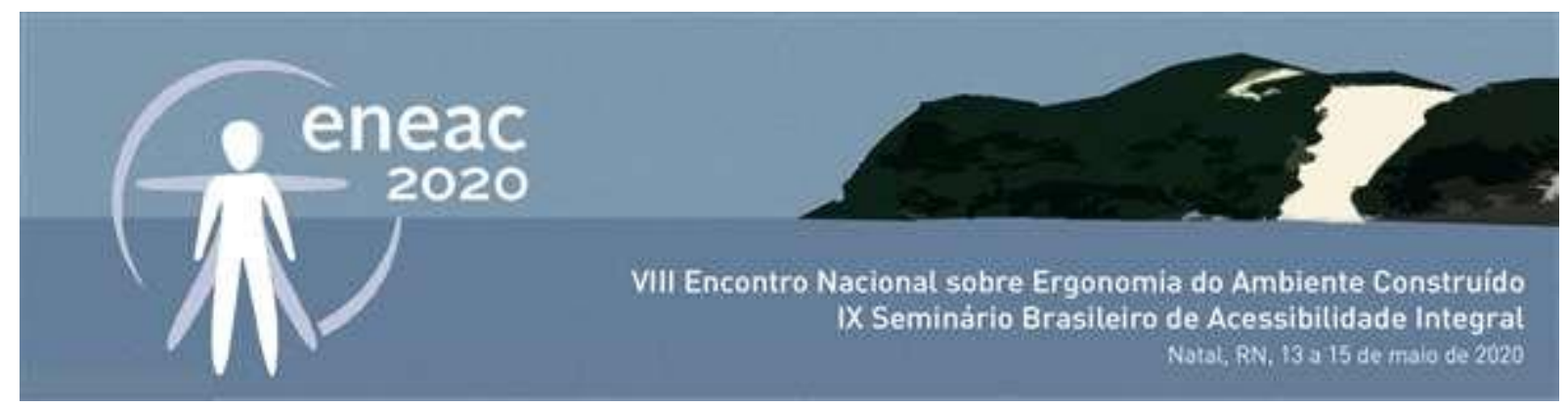

para ajudar essas pessoas a ter liberdade na navegação (por exemplo, mapas acessíveis, sistemas de navegação interna e dispositivos de computação vestíveis). No entanto, tecnologias como mapas acessíveis ou sistemas de navegação interna são insuficientes para preencher a lacuna de navegação independente, pois são necessárias informações adicionais (obstáculos, barreiras e acessibilidade). Para promover a navegação interna e criar um melhor uso das tecnologias para as pessoas com deficiência visual, é essencial entender os fatos e problemas reais que elas experimentam e quais comportamentos e estratégias eles usam para superar quaisquer problemas; essas são as preocupações que levaram a este estudo. Ao todo, 30 pessoas com deficiência visual e 15 especialistas foram recrutados para dar uma entrevista sobre o comportamento e as estratégias usadas para navegar em espaços internos e espaços públicos, por exemplo, universidades, hospitais, shoppings, museus e aeroportos. Os resultados deste estudo revelam que navegar dentro de edifícios e espaços públicos cheios de características desconhecidas é muito difícil ao tentar pela primeira vez por várias razões, reduzindo sua confiança na navegação independente.

\section{CONSIDERAÇÕES FINAIS}

Os estudos apresentados nesta revisão sistemática reiteram a importância do uso da tecnologia assistiva para uma maior apropriação do espaço, e consequentemente um melhor uso deste. Tornar possível a mobilidade e acessibilidade do espaço urbano para os cegos é um desafio factível, mas demanda um olhar diferenciado, onde o usuário, neste caso, o cego, deve ser sempre assistido com muita atenção e fazer parte de todo o processo de evolução da pesquisa.

As pesquisas apontam que a inserção de mapas táteis e aplicativos de mobilidade visam a criação de alternativas e meios que atendam um maior espectro de usuários cegos. Neste sentido, percebe-se a importância de se estudar o perfil destes usuários e atender de forma particular cada peculiaridade apreendida.

Assim, durante o processo de investigação desta revisão sistemática, foram consideradas essas duas ferramentas, delimitando um universo específico de tecnologias. Em um primeiro momento percebeu-se que a utilização das duas ferramentas otimiza o processo, onde o uso de mapas táteis tem o papel fundamental na formação da representação espacial do meio urbano, enquanto, o aplicativo de mobilidade simplifica podendo otimizar as rotas.

A partir do entendimento do processo de evolução das pesquisas, ratifica-se que atualmente existe um número considerável de estudos que abrangem esta temática. Contudo, não se esgota a possibilidade de implementação e otimização destas ferramentas, sendo de extrema relevância o contínuo processo de investigação e enriquecimento deste assunto.

\section{REFERÊNCIAS}

ALMEIDA, Maria deFatima XM; MARTINS, Laura B.; LIMA, Francisco J. Analysis of wayfinding strategies of blind people using tactile maps. Procedia Manufacturing, v. 3, p. 6020-6027, 2015.

BALATA, Jan; MIKOVEC, Zdenek; SLAVIK, Pavel. Landmark-enhanced route itineraries for navigation of blind pedestrians in urban environment. Journal on Multimodal User Interfaces, v. 12, n. 3, p. 181-198, 2018.

Cochrane Handbook, Disponível em : <http://handbook.cochrane.org> Acesso em 2 de abril de 2019.

CUTURI, Luigi F. et al. From science to technology: Orientation and mobility in blind children and adults. Neuroscience \& Biobehavioral Reviews, v. 71, p. 240-251, 2016. 


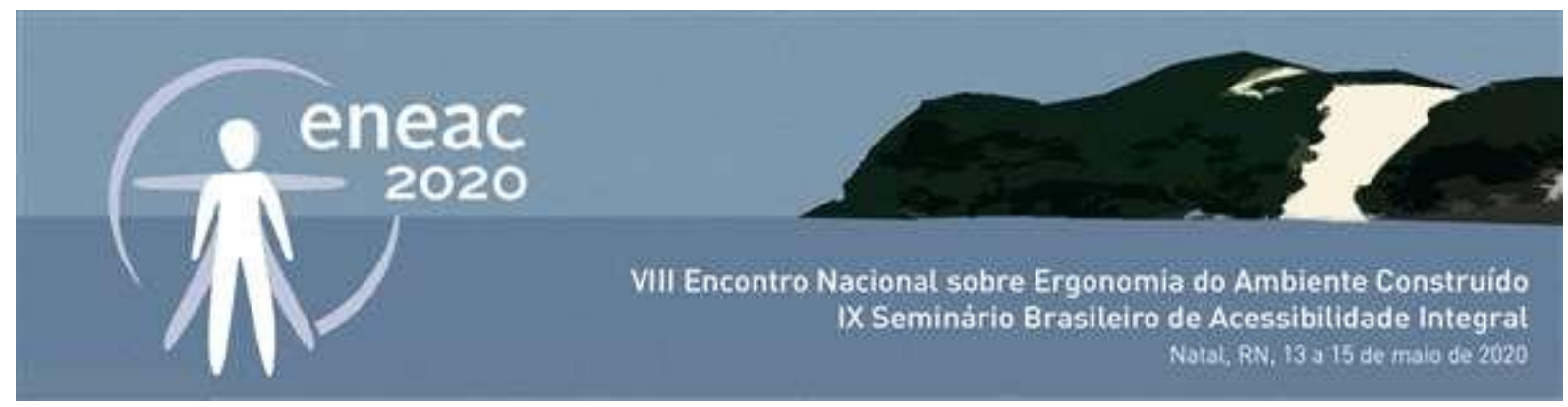

GIUDICE, Nicholas A. et al. Evaluation of an accessible, real-time, and infrastructure-free indoor navigation system by users who are blind in the mall of america. Journal of Visual Impairment \& Blindness, v. 113, n. 2, p. 140-155, 2019.

GORI, Monica et al. Corrigendum: Shape Perception and Navigation in Blind Adults. Frontiers in psychology, v. 8, p. 882, 2017.

HERSH, M.A.; JOHNSON, M.A. "Assistive Technology for visually imparied and blind people". Springer, p. 1-13.2018.

IBGE-Instituto Brasileiro de Geografia e Estatística. Censo demográfico 2010. Disponível em: http://www.ibge.gov.br/home/ estatistica/populacao/censo2010. Acesso em: 12 março. 2016.

JEAMWATTHANACHAI, Watthanasak; WALD, Mike; WILLS, Gary. Indoor navigation by blind people: Behaviors and challenges in unfamiliar spaces and buildings. British Journal of Visual Impairment, v. 37, n. 2, p. 140-153, 2019.

MELIONES, Apostolos; SAMPSON, Demetrios. Blind MuseumTourer: A system for self-guided tours in museums and blind indoor navigation. Technologies, v. 6, n. 1, p. 4, 2018.

MORASH, Valerie Starr. Movement Strategies During Haptic Search: Implications for the Learning of Blind Populations. 2014. Tese de Doutorado. UC Berkeley.

OMS - Organização Mundial de Saúde. Classificação internacional de funcionalidade, incapacidade e saúde. São Paulo: Edusp; 2003.

PIGEON, Caroline et al. Cognitive load of walking in people who are blind: Subjective and objective measures for assessment. Gait \& posture, v. 67, p. 43-49, 2019.

\section{PORTER, C. A. Psychological Implications: Psychological adjustment to visual impairment.2002.}

RABASCO, F. P. Sociedad de la información y las personas ciegas. In: CASTELLANOS, A. R. et al. (org.). Prototipos: Lenguaje y representación em las personas ciegas. Cádiz: Universidad de Cádiz, 2009, p. 309-426

RODRIGUEZ-SANCHEZ, M. C. et al. Accessible smartphones for blind users: A case study for a wayfinding system. Expert Systems with Applications, v. 41, n. 16, p. 7210-7222, 2014.

SANCHES, Emilia Christie Picelli; MACEDO, C. M. S.; BUENO, J. Imagens táteis tridimensionais: um modelo para a tradução tátil a partir de imagens estáticas bidimensionais. 2017.

TINTI, Carla et al. On my right or on your left? Spontaneous spatial perspective taking in blind people. Consciousness and cognition, v. 62, p. 1-8, 2018.

VERAS, L.C.S.VARGAS, O.L.DMREI. Sistema de ayuda a videntes para detectar el color y la posición de los objetos e diante estimulación táctil. VII Congresso Iberoamericano de Informática Educativa.2004.

VON DER WEID, Olivia. " A Urca é o paraíso dos cegos": mobilidade urbana, acesso à cidade e territorialização. ILUMINURAS, v. 16, n. 37, 2015. 